\title{
GADAMER: LA COMPRENSIÓN ES ANTERIOR. UNA ALTERNATIVA AL SUJETO-OBJETUALISMO
}

\section{GADAMER: UNDERSTANDING IS PRIOR AN ALTERNATIVE TO THE SUBJECT-OBJECT DICHOTOMY}

\section{Carmen Segura Peraita ${ }^{1}$ \\ Universidad Complutense (España)}

Recibido: 21-07-2014

Aceptado: 06-09-2014

Resumen: La filosofía hermenéutica representada por Heidegger y Gadamer ha llamado la atención sobre el comprender, destacando su prioridad ontológica sobre la intelección, que tiene un carácter derivado respecto de aquella. A la vez, ambos pensadores destacaron el carácter situado, histórico y finito de la razón. Al hacerlo mostraron su disconformidad con el modelo moderno de ciencia y conocimiento, dependiente de una metafísica representacionista que condujo a una visión sujeto-objetualista del conocimiento y de nuestro acceso al mundo. Tal visión genera problemas en realidad artificiales, como es el caso del debate realismo-idealismo. Este trabajo muestra la posibilidad de su disolución en la idea gadameriana de pertenencia.

Palabras-clave: comprender, pertenencia, sujeto-objetualismo, anti-realismo, acontecer.

\begin{abstract}
The hermeneutical philosophy represented by Heidegger and Gadamer has brought attention to the issue of understanding, highlighting its ontological priority over intellection, which has a derived character with respect to the former. At the same time, both thinkers emphasized the situated, historical and finite character of reason. In so doing they demonstrated their non-conformity with the modern model of science and knowledge, which is dependent on a representationalist metaphysics that has led to a subjectobject dichotomy regarding both knowledge and our access to the world. This vision gives rise to problems that are, in reality, artificial, as is the case with the realism-idealism debate. This article shows that the Gadamerian idea of belonging makes the dissolution of this problem possible.

Key-words: understanding, belonging, subject-object dichotomy, anti-realism, event.
\end{abstract}

[1] (csegura@filos.ucm.es) Profesora Titular del Departamento de Filosofía Teorética de la Facultad de Filosofía (Universidad Complutense de Madrid). 


\section{Introducción}

La que se podría denominar hermenéutica ontológica -representada principalmente por Heidegger y Gadamer- ha introducido modificaciones sustanciales en la manera de concebir la filosofía, pero ante todo, ha modificado nuestra visión de mundo y del ser humano. Por lo que se refiere al conocimiento, la intelección, lo que ambos pensadores entienden es que ésta es un fenómeno derivado de otro anterior y más fundamental: la comprensión. Comprender es el modo de ser del Dasein, del ser humano. En esto consiste su existencia, que es posibilidad abierta. Aunque ambos pensadores han expresado y modulado de diferente manera esta idea fundamental, coinciden en lo esencial. Mientras que la comprensión se sitúa en el nivel ontológico, la intelección lo hace en el epistemológico o gnoseológico. La comprensión es originaria, la intelección es derivada. Y es que el ser humano -entendido como ser-en-el-mundo- es eminentemente práxico y no teórico. Su existencia consiste en ser ya en el mundo comprendiéndolo, relacionándose con él de manera práctica, sin una previa mediación intelectual y, con Gadamer, en el medio del lenguaje. Claro está que la teoría es una de la formas, derivadas, en que el Dasein, puede tener que ver con las cosas, pero no es la primera ni la constituyente.

Ni Heidegger ni tampoco Gadamer rechazan ni menosprecian la teoría, tan sólo le encuentran su lugar propio y, a hacerlo, ofrecen una visión más lograda, al menos a mi juicio, de la existencia humana, del conocimiento y del mundo. La crítica de Heidegger al primado clásico de la teoría en la metafísica occidental tiene su comienzo en el denominado Natorp Bericht y se extiende hasta el final de su carrera, aunque con modificaciones notables en la manera de realizarla. Algunos hitos destacables los encontramos en el curso El Sofista, en Ser y tiempo, en "La época de la imagen del mundo", en la "Carta sobre el humanismo" y en "Tiempo y ser". Heidegger entiende que la intelección y su primado son un producto típico de la metafísica, que es representacionista por su propia constitución y que desde Platón entiende la verdad como adaequatio. También su discípulo Gadamer critica en Verdad y método el intelectualismo occidental, en particular en su versión moderna, aquella que reduce el conocimiento al modelo de la ciencia cartesiana y la verdad al método. Por otra parte, sus numerosos escritos sobre filosofía griega, tienen la misma orientación y se encaminan a una recuperación de algunas intuiciones originales que se perdieron posteriormente.

Frente al ideal de un conocimiento objetivo y universal, de una verdad absoluta y atemporal, ambos destacan la finitud e historicidad del ser humano. Ambos ponen en primer plano su carácter situacional y con él el de la razón. Por eso Gadamer reivindica "un saber no separado del ser". Con Heidegger y Gadamer, la finitud, la muerte, el tiempo y la historia pasan a un primer plano y no precisamente como condicionantes "negativos" que haya que superar, sino 
como caracteres irrenunciables y constituyentes de nuestro ser-en y comprender el mundo. Esas notas adquieren, por tanto, una dimensión positiva.

A mi juicio, la idea heideggeriana de "situación hermenéutica" da buena cuenta de esto que se acaba de apuntar y articula de manera determinante el modo en que ambos pensadores entienden y despliegan nuestro ser comprensor del mundo en el que ya siempre y de antemano estamos. En realidad, la idea de "situación hermenéutica" constituye un mentis a la idea clásica y moderna de intelección, un mentis que va también contra los ideales ilustrados de objetividad y progreso indefinido de la razón.

Esa idea expresa, resumidamente, que el ser humano-que es ya desde siempre comprensor- está hermenéuticamente situado; es decir, que se encuentra bajo una determinada interpretación (fruto de la herencia histórica y, así, también de los condicionamientos sociocultares, etc.) de la que no puede escapar porque le constituye en su ser mismo. Es decir, todo ser humano se encuentra en una determinada situación de interpretación. De acuerdo con el joven Heidegger, dicha situación está determinada por un punto de mira, una dirección y un horizonte de la mirada, de tal manera que la interpretación que se quiera realizar en cada caso es posible en la medida en que desde la óptica de esas tres coordenadas se aclara la situación desde la que se realiza esa interpretación ${ }^{2}$. Esto es tanto como insistir en nuestro carácter situacional y finito declarando la imposibilidad de un conocimiento libre de pre-juicios; es decir de pre-comprensiones o anticipaciones de sentido. Dicho de otra manera, esta vez con Gadamer, esto implica el carácter prejuicioso de nuestra comprensión y de nuestra razón.

Ahora bien, hemos de entender bien de qué se habla cuando se hace de los prejuicios. Acostumbramos- también por los usos que de esta palabra hacemos en el lenguaje ordinario- a tener una idea muy negativa de los mismos, entendiendo que nublan e incluso impiden la comprensión. Pero cuando el autor de Verdad y método desvela su inevitable presencia les otorga un carácter y un sentido bien distinto. Porque no podemos no tener prejuicios, ya que siempre nos movemos en un determinada comprensión -una comprensión de término medio, diría quizá Heidegger-; es decir, porque siempre que nos planteamos una pregunta o abordamos un problema lo hacemos desde algo anterior, que lo hace posible y que lo sitúa en un determinado horizonte. Las preguntas no se podrían plantear y la ciencia no podría avanzar si no fuera porque ya contamos con un haber previo, que es finito, situado e histórico, pero que es un haber al fin y a la postre. Pues bien, ese haber -que ha de ser modificado e incluso sustituido en el decurso de la investigación-es lo que hace posible la profundización en el conocimiento y con ella el progreso, aunque siempre limitado y variable. Por otra parte, ya se ha mencionado también que ese haber no es sino una

[2] Heidegger, M., Natorp Bericht, GA 61, p. 1.

THÉMATA. Revista de Filosofía, Nº50 julio-diciembre (2014) pp.: 229-245 doi: 10.12795/themata.2014.i50.11 
anticipación de sentido, pues de lo contrario, ¿cómo buscaremos aquello que ignoramos totalmente? ${ }^{3}$.

Que el ser-en-el-mundo está hermenéuticamente situado implica que es esencialmente temporal histórico. El tiempo no es un recipiente ni una medida homogénea sino la misma esencia de nuestra existencia. Por tanto, también nuestra comprensión e intelección, nuestras teorías, lo son. La historicidad caracteriza la comprensión y al hacerlo delata nuestra finitud que tiene su expresión más acabada en la muerte.

Al llevar al primer plano nuestra finitud, Heidegger y Gadamer se oponen frontalmente al intelectualismo que ha dominado desde el principio toda la metafísica occidental, imponiendo un duro correctivo a su desmedida ansia de absoluto. Con Sócrates, Platón y Aristóteles se impone la sophrosine (que es también aquella diosa que logro escapar de los males desatados tras abrirse la caja de Pandora). Tanto Heidegger como Gadamer entendieron que en el origen de la metafísica moderna-aquella que se acostumbra a entender fundada por Descartes- se encontraba el germen de la antigua, aunque de alguna manera desnaturalizado. Lo cierto es que la tradicional comprensión de la verdad como rectitudo, adaequatio, alcanza su paroxismo en la Modernidad, en aquel momento en que el conocimiento se entiende y establece en términos de representación (Vor-stellung) y en el que se establece un hiato entre sujeto y objeto. Perdido el sentido de la intencionalidad (con Descartes) el sujeto se autoconstituye como una conciencia ab-soluta y se autoclausura, puesto que se entiende a sí mismo como fundamentum absolutum inconcusum veritatis. Y frente a él el ob-jectum, que no es ya la cosa (pragma) sino lo congnoscible. Así queda "desnaturalizado" el mundo y el ser-en-el-mundo; así queda anulada la posibilidad de plantear siquiera la pregunta por las condiciones de posibilidad del comprender. De este modo, más que nunca, se hace necesario establecer un puente, cuando antes no lo había, entre el yo y el mundo. Ahora, entre el sujeto y el objeto.

En realidad se trata de un artificio, de una construcción que merma las posibilidades del conocimiento, de la verdad y de la misma ciencia. En oposición a él, Gadamer elabora las nociones de acontecer de la comprensión y de pertenencia. Por lo que a esta última se refiere, bastará por el momento con apuntar, puesto que será objeto de un desarrollo más detenido en este trabajo, que expresa la relación mutua del ser y el lógos, aquella que destacó Parménides en el comienzo de la metafísica occidental, afirmando que "lo mismo es pensar y ser" y que Heidegger tematizó más tarde como co-pertenencia de Dasein y ser. Pero frente a las lecturas tópicas de la sentencia parmenídea, la nueva "ontología fenomenológico-hermenéutica" de Heidegger y también de Gadamer, que es dinámica, realiza un interpretación histórica. Es posible que ésta no sea

[3] Cf., Menón, 80d.

THÉMATA. Revista de Filosofía, Nº50 julio-diciembre (2014) pp.: 229-245 doi: 10.12795/themata.2014.i50.11 
neutral, ni siquiera "objetiva", pero da cauce a ese aspecto central no recogido por el pensamiento anterior: el que tiene que ver con el ya mencionado carácter histórico de la comprensión y el conocimiento. De ahí la idea de que la comprensión acontece, también porque la hermenéutica no participa de la idea de que haya algo semejante a "los seres en si". Por el contrario, la verdad se devela y constituye en medio de un proceso similar al de una verdadera conversación. Gracias a ella al final surge algo que antes no era. Por eso Gadamer concede una importancia tan central a la pregunta, que declara anterior al enunciado teórico, puesto que, como sostiene, todo enunciado no es sino la respuesta a un pregunta, que es siempre anterior. Una pregunta que abre y que tiene una determinada dirección y orientación. Gadamer, el discípulo de Sócrates, sabe bien que el diálogo es el camino, el método, para buscar la verdad; pero se trata de un diálogo infinito, que no acaba nunca y del que nunca obtendremos al final una respuesta cerrada y definitiva.

La conocida crítica de Heidegger y Gadamer a la ciencia moderna y a su ideal de método, se dirige de manera preeminente a su comprensión sujeto-objetualista del conocimiento. Con las ideas centrales de que la comprensión es anterior, de ser-en-el-mundo y de pertenencia se pueden conjurar las distorsiones a que aquella da lugar. En particular, se puede afrontar de manera eficaz el viejo debate "realismo-idealismo". Un debate tan clásico como innecesario si, desde una visión fenomenológico-hermenéutica, como es la de Heidegger y también la de Gadamer, se entiende que ser es aparecer. Afirmación está que entronca de nuevo con la sentencia parmenídea, aunque constituya una "variación fenomenológica" de la misma.

En las líneas que siguen me ocuparé de desarrollar algo más detenidamente algunas de las ideas gadamerianas expresadas aquí tan sucintamente. En particular desarrollaré lo que tiene que ver con la anterioridad de la comprensión sobre la intelección y me centraré en la noción de pertenencia [Zugehörigkeit]. Su tratamiento me permitirá abordar lo relativo al sujeto-objetualismo moderno y al debate realismo-idealismo a él asociado.

\section{El debate realismo-idealismo}

Sabemos que el autor de Verdad y método centra su reflexión sobre la experiencia hermenéutica del acontecer de la comprensión en las ciencias del espíritu, pero también que desde ahí y teniendo siempre como centro al lenguaje, extrapola sus conclusiones a una comprensión general del ser y de la verdad, de las ciencias en su conjunto y del conocimiento humano. Al hacerlo ofrece una visión que, ya lo hemos dicho, no es ni realista ni idealista, porque constituye una recusación del sujeto-objetualismo aquel que, en última instancia de pábulo a dichas posiciones. Se trata de una comprensión que las deja a ambas de lado y que supone un acercamiento más ajustado al problema que tanto el realismo como el idealismo quieren afrontar, aunque en realidad, lo crean. 
Pero es necesario subrayar que lo que importa a la hermenéutica filosófica no son los actos cognoscitivos derivados, como el juicio o los razonamientos. Ella atiende más bien a las condiciones ontológicas de posibilidad del comprender en general, que es anterior a cualquier realización concreta del mismo (de todo y cualquier acto de inteligir, razonar, demostrar, etc.). La razón es que, tal como Gadamer sostiene, comprender es la forma original de realización del ser humano, su modo propio de ser, que es poder ser o posibilidad. Sencillamente existir es comprender (y toda comprensión es lingüística) ${ }^{4}$. Por esta razón Gadamer se plantea radicalmente el problema de comprender. Ahora bien, hacerlo, como ha recordado Escudero, requiere situarse expresamente más acá de la contraposición entre el 'realismo' y el 'idealismo", ya que la tesis de que las condiciones de posibilidad del comprender "no pueden situarse unilateralmente ni en el sujeto que comprende ni en el objeto comprendido" lo impide. Ahora bien, precisamente por lo que se acaba de decir, el tratamiento hermenéutico de la comprensión es determinante a la hora de clarificar cómo es nuestro acercamiento al mundo en cada caso ${ }^{6}$. También por eso puede decir algo significativo para el debate realismo-idealismo.

Pues bien, Hilary Putnam ha resumido de un modo acertado la comprensión del mundo que es propia del realismo metafísico:

El mundo consta de una totalidad fija de objetos independientes de la mente. Hay exactamente una descripción verdadera y completa de 'cómo es el mundo'. La verdad supone una especie de relación de correspondencia entre palabras o signos mentales y cosas o conjuntos de cosas externas"7.

De ambas caracterizaciones obtenemos los siguientes asertos: 1) existen entidades con sus correspondientes propiedades que son independientes del sujeto. 2) La verdad se entiende como adecuación o correspondencia ${ }^{8}$.

[4] "Comprender deja de ser un acto de intelección para convertirse en el modo de ser por el que el hombre se encuentra siempre existiendo en un horizonte de posibilidades a las que está referido y en virtud del que existir es siempre poder-ser". Rodríguez, R., "Hermenéutica y ontología: ¿Cuestión de método?”, Métodos del pensamiento ontológico, Síntesis, 2002, pp. 235-254, p. 257.

[5] Escudero, "Hermenéutica de la verdad", en Oñate, T. et alia (eds.), Hans-Georg Gadamer, Ontología estética y hermenéutica, Dykinson, Madrid, 2005, p.150. Citando a Luís Sáez Rueda, Movimientos filosóficos actuales, Trotta, Madrid, 2001, pp. 181-182.

[6] "La comprensión permite el acceso a la pre-estructura o ámbito originario de la verdad que determina todo lo que el ser humano es. Estructura originaria que legitima todo conocimiento, no sólo el de las ciencias del espíritu, sino incluso el científico experimental [Todas las formas de racionalidad] surgen de esa estructura originaria o verdad a la que se accede por la comprensión hermenéutica, por esa comprensión participativa que es un comprenderse”. Lozano Díaz, V., Hermenéutica y fenomenología, EDICEP, Valencia, 2006, 99.

[7] Putnam, Hillary, Reason, Thruth and History, Cambridge University Press, Cambridge, 1981, p. 49. [Vers. cast., Razón, verdad e historia, Tecnos, Madrid, 1988.

[8] Cuando decimos que algo es verdadero, en fin, es como si dijésemos cómo están y estarían las cosas incuso si nosotros mismos, que pronunciamos y escuchamos esa frase, no existiésemos

THÉMATA. Revista de Filosofía, №50 julio-diciembre (2014) pp.: 229-245 doi: 10.12795/themata.2014.i50.11 
Pues bien, la hermenéutica filosófica, desarrollada y representada por Hans-Georg Gadamer, invalida dichas tesis y se sitúa situada más allá, o más acá, del debate entre realismo e idealismo. De hecho, se acostumbra a ver en dicha hermenéutica una posición antirealista, aunque más bien habría que detectar, como veremos a continuación, una posición "antirepresentacionista". En este sentido, Chiurazzi afirma certeramente que la hermenéutica,

\begin{abstract}
posee su propia concepción de la realidad, que no es de hecho la del realismo metafísico: la idea de la existencia de un mundo en sí, del todo independiente de nuestra experiencia, sino aquélla propia del realismo físico, que no tiene ya nada que ver con la esencia, sino más bien con la existencia y con relaciones entre existencias; una realidad, por tanto, dinámica. Este concepto de realidad es el fundamento, no del anti-realismo, sino del anti-representacionalismo que caracteriza sin duda a la hermenéutica filosófica, pero, no por casualidad, también a una gran parte de la física contemporánea ${ }^{9}$.
\end{abstract}

Lo cierto es que la esencial estructura del ser-en-el-mundo niega la posibilidad de inclusión de la hermenéutica en ninguna de las dos posiciones antagónicas que se acaban de mencionar, realismo e idealismo. Otro tanto sucede con la central idea de que ser en aparecer, que muestra la vinculación esencial entre fenómeno y lógos, tal como quedó establecida en el parágrafo 7 de la Introducción a Ser y tiempo. Lo cierto, para el caso que nos ocupa aquí más específicamente, es que la hermenéutica filosófica resulta incompatible con las tesis realistas. Veámoslo.

En primer lugar, la misma idea de "mundo", en torno a la que gira la caracterización de Hilary Putnam, ya resulta problemática en sí misma. Y más aún lo es la de "mundo en sí", al menos desde la hermenéutica gadameriana según la cual el mundo está constituido lingüísticamente, de manera que sólo podemos llegar a tener "acepciones del mundo". No es éste el momento para profundizar en la central cuestión de la lingüisticidad de nuestra experiencia. Tan sólo quiero poner el acento en la noción de "acepción del mundo", para precisar que, de acuerdo con Gadamer, "la multiplicidad de tales acepciones del mundo no significa relativización del 'mundo'. Al contrario, lo que el mundo es no es nada distinto de las acepciones en las que se ofrece"10. En resumen, el mundo es su acepciones, lo que no implica, naturalmente, que no haya un mundo, en el sentido de la naturaleza, los artefactos, etc., que sea independiente de los seres humanos. Significa, simplemente, la coherente negativa a aceptar la viabilidad y operatividad de la noción de "mundo en sí". Porque como ha subrayado Wachterhauser, lo que conocemos es 'el mundo' en toda su compleja variedad. No son precisamente los objetos naturales ni sus relaciones según

de hecho". Chiurazzi, G., "La realidad de la hermenéutica", en Oñate et alia (eds.), Acontecer y comprender, Dykinson, Madrid, 2012, p. 85.

[9] Ib., pp. 83-84.

[10] Gadamer, H.-G., Verdad y método, Sígueme, Salamanca, 1997, p. 536.

THÉMATA. Revista de Filosofía, №50 julio-diciembre (2014) pp.: 229-245

doi: 10.12795/themata.2014.i50.11 
leyes las que constituyen el mundo, sino otra gente, otras culturas, tradiciones, lenguaje, normas, textos, instituciones, prácticas, etc., las que constituyen el mundo como una realidad cognoscible y plena de significado ${ }^{11}$. Sobre esto tendré que volver más adelante.

En segundo lugar, por lo que se refiere a la existencia de entidades independientes del sujeto, hay que destacar que en la hermenéutica filosófica, primero en la de Heidegger y después también en la de Gadamer, encontramos una superación brillante de la metafísica de la sustancia. Esto es, la hermenéutica niega que la realidad esté constituida por entidades cuya esencia sea inmutable y permanente (ajena al tiempo y la historia); una postura que ofrece los fundamentos para las posiciones realistas, como acabamos de ver. Ahora bien, si la realidad ya no está constituida por unidades monádicas inmutables, por sustancias, entonces lo está por relaciones, estructuras y los efectos a que estos dan lugar. El acontecer es el modo de darse lo que es. Esto a lo que me estoy refiriendo se encuentra emparentado con la central noción gadameriana de "conciencia de la historia efectual", sobre la que también volveré más adelante. A la vez, la misma hermenéutica invalida la moderna metafísica del sujeto. Es decir, destierra la posibilidad de existencia de una conciencia autónoma que pudiera constituir o construir la realidad desde sí misma, ya que, de acuerdo con la fundamental noción de pertenencia (que articula este trabajo) "lo primario es la relación"12. Y es que, antes del sujeto hay un "hacer de la cosa misma" que éste padece ${ }^{13}$.

En tercer lugar, por lo que tiene que ver con la verdad, la hermenéutica filosófica rechaza aquella concepción adecuacionista que tiene su apoyo en las metafísicas ya descartadas y que encuentra su sustento en una visión representacionista del conocimiento. Frente a esta concepción clásica, la hermenéutica fenomenología -que tiene su arraigo en los desarrollos de Heidegger y que se despliega a lo largo de la obra de Gadamer-, sostiene que ser es aparecer; es decir, que los fenómenos se constituyen en cada una de sus manifestaciones y que, como ya se ha apuntado, aparecer es acontecer, de manera que la experiencia es dinámica. Tal acontecer se resuelve en una cuestión de pertenencia ${ }^{14}$ : una vez más, también por lo que se refiere a la verdad, "lo primario es la relación"; dicho de otro modo "la relación del todo es más originaria que la de las partes". Por esta razón, la comprensión no puede ser copia ni preproducción de una realidad fija.

En lo que sigue desarrollaré con algo más de detalle las ideas expuestas aquí. Para ello habré de centrarme en la central noción de pertenencia.

[11] Cf. Wachterhauser, B., "Getting it Right: Relativism, Realism and Thruth", en Dostal, R. J. (ed.), The Cambridge Companion to Gadamer, Cambridge University Press, 2002, p. 73.

[12] Cf., Gadamer, H.-G. Verdad y método, op. cit., p. 463.

[13] Cf. Ib., p. 478.

[14] Cf. Garcia Santos, "Historicidad y pertenencia en la hermenéutica de Gadamer: dos problemas y ¿una solución?”, en Oñate. T., et alia (eds.), Acontecer y comprender, op. cit., p. 140.

THÉMATA. Revista de Filosofía, №50 julio-diciembre (2014) pp.: 229-245 doi: 10.12795/themata.2014.i50.11 


\section{La noción de pertenencia (Zugehörigkeit).}

\subsection{La pregunta hermenéutica fundamental por las condiciones de posibilidad del comprender}

El estudio de las condiciones de posibilidad de comprender, realizado por Gadamer, se remite sin duda a la pregunta kantiana por los límites de nuestra experiencia posible. Al afrontarla Gadamer encuentra el límite en nuestra situación hermenéutica, esa que está necesariamente constituida por los pre-juicios, y que nos hace entender que el sujeto no es sólo lo que condiciona sino que también él es condicionado puesto que está situado históricamente. Así pues, los prejuicios y la historicidad de la comprensión delimitan el alcance de nuestra experiencia posible.

Por lo que se refiere a la estructura prejuiciosa de la comprensión bastará con recordar que el comprender funciona especialmente mediante los pre-juicios, de tal manera que, aunque seamos conscientes de su presencia no podemos librarnos de ellos ${ }^{15}$. Pero dicha presencia no es negativa, como quería la Ilustración. "Los prejuicios son, en efecto, las anticipaciones de sentido que hacen posible la comprensión y que hay ante todo que entender en su función positiva de condiciones del comprender"16. Es decir, comprendemos porque tenemos prejuicios, o lo que es lo mismo, ellos son condiciones posibilitantes de la comprensión. Puesto que somos finitos y situados, no es posible la ausencia de punto de vista o de perspectiva, no es posible, en definitiva, un conocimiento libre de supuestos.

Para comprender algo tenemos que estar instalados en un horizonte significativo, a partir del cual pueda en primer lugar aparecer el objeto de nuestra comprensión, pueda interesarnos o arrastrarnos hacia él, y pueda en segundo lugar empezar a ser comprendido, produciéndose la conciencia propia del sujeto como parte de la totalidad histórica ${ }^{17}$.

“Cómo buscaremos aquello que ignoramos completamente?”, pregunta Menón a Sócrates en el diálogo homónimo. Sabemos que la respuesta del maestro es la teoría de la reminiscencia. Pero la del socrático Gadamer sería otra (aunque quizá no tan alejada como podría parecer en un primer momento): no podemos buscar lo que desconocemos totalmente, a no ser que haya una precomprensión, un horizonte y unas anticipaciones de sentido. La pregunta del que busca y se interroga se encuentra a la vez situada en un horizonte y es abierta; si es una auténtica pregunta se desconoce la respuesta, pero la búsqueda está guiada por nuestras precomprensiones a la vez que "por el hacer de

[15] Cf., Rodríguez, R. "Hermenéutica y ontología”, op. cit., p. 239.

[16] Ib., p. 247.

[17] Lozano Díaz, V., Hermenéutica y fenomenología, op. cit., p. 96.

THÉMATA. Revista de Filosofía, Nº50 julio-diciembre (2014) pp.: 229-245 doi: 10.12795/themata.2014.i50.11 
la cosa misma". La conciencia de la historia efectual, elemento nuclear de la hermenéutica filosófica gadameriana es la de estar expuestos de tal manera a los efectos de la historia que éstos forman parte de nuestro ser.

En la realidad no es la historia la que nos pertenece, sino que somos nosotros lo que pertenecemos a ella. Mucho antes de que nosotros nos comprendamos a nosotros mismos en la reflexión, nos estamos comprendiendo ya de una manera autoevidente en la familia, la sociedad y el estado en que vivimos. La lente de la subjetividad es un espejo deformante. La autorreflexión del individuo no es más que una chispa en la corriente cerrada de la vida histórica. Por eso los prejuicios de un individuo son, mucho más que sus juicios, la realidad histórica de su ser ${ }^{18}$.

Estas últimas palabras de Gadamer, vinculan de manera sólida la estructura prejuiciosa con el carácter histórico de la comprensión, que era el segundo aspecto que debíamos abordar. Para hacerlo, teniendo en cuenta el propósito que guía este trabajo, resulta útil que me remita al trabajo de Wachterhauser, "Getting it Right: Relativism, Realism and Thruth"19, sintetizando aquí algunas de sus observaciones.

Este excelente estudioso de Gadamer ha insistido, apoyándose en las aportaciones de Hilary Putnam, en que cada avance teórico tiene su propia historia. Para mostrarlo recuerda que incluso términos como "electrón" tienen su propia historia conceptual, que se va modificando a lo largo de la investigación. De esta manera, entiende que la pretensión de probar la adecuación a algo inmutable resulta injustificada. Por otra parte, Wachterhauser acude también a aquellos sociólogos de la ciencia que han puesto de manifiesto cómo toda exigencia de verdad está condicionada por valores e intereses de los investigadores y de la comunidad científica. Esto plantea la cuestión más amplia acerca de si un objeto puede ser independiente del lenguaje, y de esos valores e intereses. La respuesta es que toda exigencia de verdad es "relativa" al punto de vista de la interpretación realizada por los investigadores. Así pues, todo apunta, como subraya nuestro investigador, a que la "pura" teoría no es tan pura, si es que la pureza significa ser conceptualmente independiente de nociones normativas implícitas en el éxito intelectual. En consecuencia, se ha de concluir que no hay hechos libres de valoración o conceptualmente neutrales, hechos a los que nuestra interpretación pueda simplemente corresponder. En definitiva, siempre hay interpretación ${ }^{20}$. Es el mismo Gadamer quien lo sostiene, cuando afirma que,

el único dato real es que comprendemos algo como 'algo'. La fundamentación del conocimiento no puede evitar en el ámbito de las ciencias naturales la consecuencia hermenéutica de que la realidad 'dada' es inseparable de la interpretación”"1.

[18] Gadamer, H.-G., Verdad y método, op. cit., p. 344.

[19] Cf., Wachterhauser, B., "Getting it Right: Relativism, Realism and Thruth", op. cit.

[20] Cf., Ib., pp. 53-4.

[21] Gadamer, H.-G., “Texto e interpretación”, Verdad y método II, Sígueme, Salamanca, 1994, p. 327.

THÉMATA. Revista de Filosofía, Nº50 julio-diciembre (2014) pp.: 229-245 doi: 10.12795/themata.2014.i50.11 
Sabemos que, al realizar esta afirmación, Gadamer no está declarando algo insólito ni novedoso. Por el contrario, está rememorando y actualizando algo bien sabido desde los pensadores griegos, desde Platón, los sofistas y Aristóteles: que algo siempre aparece como algo (estructura ti katá tinós). En eso precisamente consiste la posibilidad de juicio y de la verdad. Se trata de una comprensión bien asentada y que se puede ver recogida, por ejemplo, en el Peri hermenéias o en el libro de las Categorías. Algo se dice siempre como algo porque aparece como algo, y el lenguaje, la comunicación y la verdad son posibles por este sencillo hecho. Hecho que, una vez evidenciado, nos facilita entender mejor que la comprensión es un acontecer hermenéutico, que, como también sostiene Gadamer, "es al mismo tiempo apropiación e interpretación" ${ }^{22}$. Ahora bien, como ha aclarado Ramón Rodríguez, “

Que el acto de comprensión sea una interpretación no significa que no haya un efectivo darse de las cosas y un componente de clara pasividad del sujeto, sino que esa donación de sentido - el que lo que se dé sea inteligible- es precisamente posibilitado porque ya está rigiendo un campo de significación, que no es introducido por el propio acto cognoscitivo (la 'intuición'), y gracias al cual las cosas aparecen como 'ellas mismas'. El conocimiento, incluso el más familiar, es interpretación porque el sentido de lo dado se constituye a partir de la significación vigente en el horizonte de posibilidades que es el mundo"23.

Una vez más, entendemos que la comprensión es posible por los prejuicios legítimos, que no son otra cosa que esas precomprensiones que se ha transmitido históricamente y que anteceden y posibilitan todo acto intelectual metódico ${ }^{24}$.

Gadamer ha mostrado que toda pretensión de verdad es histórica porque está situada en una determinada tradición de investigación, de manera que esa tradición proporciona el contexto normativo para la misma. En este sentido -apunta también Wachterhauser- la tradición determina cuestiones tan decisivas como qué cuestiones son las más importantes, qué tiene prioridad para una particular comunidad de investigadores en un determinado tiempo, qué instrumentos conceptuales son los aceptables para intentar resolver esa cuestión, etc. ${ }^{25}$. Lo cierto es que no somos plenamente libres, como agentes racionales, pues suponer lo contrario implicaría que somos capaces de juzgar la totalidad de la naturaleza y las normas que gobiernan el conocimiento, lo que exigiría que éstas fueran transparentes para nosotros mismos, lo que no es el caso. Así pues, el modelo de autonomía racional cartesiano y kantiano es rechazable, debido a

[22] Gadamer, H.-G., Verdad y método, op. cit., p. 555.

[23] Rodríguez, R., "Hermenéutica y ontología", op. cit., p. 259.

[24] "Un prejuicio legítimo no es otra cosas que una precomprensión transmitida históricamente que precede a la inteligencia, la cual se logra después mediante una exposición metodológica, y que muestra precisamente la existencia de ese estado original natural o primer horizonte previo de experiencia general del mundo al que Gadamer llama verdad”. Lozano Díaz, V., Hermenéutica $y$ fenomenología, op. cit., p. 97.

[25] Cf., Wachterhauser, B., "Getting it Right: Relativism, Realism and Thruth", op. cit., p. 58.

THÉMATA. Revista de Filosofía, №50 julio-diciembre (2014) pp.: 229-245

doi: 10.12795/themata.2014.i50.11 
la finitud de nuestro conocimiento ${ }^{26}$. En consecuencia, lo que Gadamer quiere mostrar, como señala Wachterhauser, es que la autonomía coexiste con la vinculación a la tradición; sólo si estamos profundamente formados por una tradición somos capaces de modificarla en múltiples direcciones. En síntesis, el horizonte es abierto -ya lo hemos visto- pero impone condiciones a la investigación, es vinculante $^{27}$. Sin embargo, el hecho de que el conocimiento sea siempre dependiente de condiciones históricas, lingüísticas y normativas (lo que constituye un punto de partida relativo), no constituye un peligro inherente para el mismo; por el contrario, es su condición de posibilidad ${ }^{28}$.

\subsection{La pertenencia como alternativa ontológica al sujeto-objetualismo epistemológico}

Gadamer desarrolla la idea de la pertenencia "partiendo de la base de una experiencia del mundo constituida lingüísticamente"29. Ahora no nos podemos detener en este particular, pero es necesario recordarlo, pues constituye el horizonte último y fundamental de la reflexión gadameriana. De cualquier manera, lo que Gadamer quiere expresar mediante la noción de pertenencia es que quien comprende pertenece a lo comprendido, de manera que su concepción rechaza el dualismo clásico sujeto-objeto y entiende la comprensión como un momento del ser mismo; es decir, la capta y expresa en su auténtica dimensión ontológica, dejando de lado las derivaciones teóricas secundarias, que sin duda existen y contribuyen a nuestra visión del mundo, pero que dependen esencialmente de nuestro ser comprensores y se articulan desde él.

Para desarrollar esta idea de pertenencia Gadamer se acoge a la relación trascendental entre ser y verdad destacada por la metafísica clásica. Aunque sabemos que especialmente la metafísica medieval, que es la que elaboró explícitamente la teoría de los trascendentales tiene su arraigo en la griega. Platón y Aristóteles comparten más implícitamente esta visión, pero en especial hay que destacar a Parménides quien declaró en el origen la identidad de pensar y ser ${ }^{30}$. "En este pensamiento [dice Gadamer] no se hace mención de

[26] Cf., Ib., pp. 61-3.

[27] Cf., Ib., p. 63.

[28] Cf., Ib., p. 72.

[29] Gadamer, H.-G., Verdad y método, op. cit., p. 549.

[30] Como ha recordado Ramón Rodríguez, la idea de pertenencia "da cuerpo a la pretensión de la ontología de la comprensión de sustraer a la relación cognoscitiva sujeto-objeto su primacía a la hora de entender las relaciones del hombre con su mundo. El hombre no está ante el mundo, para verlo objetivamente, sino en el mundo, situado en él, penetrado por él. La relación sujeto-objeto reposa sobre un estrato más fundamental, que no es conocimiento, sino ser, y a ese ser que precede al conocimiento da forma conceptual la pertenencia". Rodríguez, R., "Hermenéutica y ontología", op. cit., p. 247.

THÉMATA. Revista de Filosofía, №50 julio-diciembre (2014) pp.: 229-245 doi: 10.12795/themata.2014.i50.11 
ningún espíritu sin mundo, con certidumbre de sí mismo y que tuviera que hallar el camino hacia el ser del mundo, sino que ambas cosas van originariamente juntas. Lo primario es la relación" 31 .

Esto, el carácter primario de la relación, es lo que se quiere destacar con el concepto de pertenencia. Pero al poner el acento en la primacía teórica y metodológica del binomio sujeto-objeto queda cancelada. Siempre de acuerdo con el autor de Verdad y método, el conocimiento es concebido como un momento del ser mismo y no como un comportamiento del sujeto ${ }^{32}$. Por tanto, "Lo que es, es por su esencia verdad (...); aquí no se parte del concepto de un sujeto que lo sea para sí y que convierta todo lo demás en objetos" ${ }^{33}$.

Es cierto que frente a los planteamientos teleológicos en los que Gadamer se apoya inicialmente, en su hermenéutica el concepto de pertenencia "no se determina ya como referencia teleológica del espíritu a la estructura esencial de los entes tal como se piensa en la metafísica". Ahora el fundamento es completamente distinto, porque, "ni la conciencia del intérprete es señora de lo que accede a él como palabra de la tradición, ni es adecuado describir lo que tiene lugar aquí como un conocimiento progresivo de lo que es"34. De lo que más bien se trata es de un acontecer que Gadamer explica ateniéndose a las ciencias del espíritu del siguiente modo.

El contenido de la tradición entra en juego y se despliega en posibilidades de sentido y resonancia siempre nuevas y siempre ampliadas de nuevo por su nuevo receptor. Cuando la tradición vuelve a hablar, emerge algo que es desde entonces, y que antes no era" (...). No hay un ser en sí que se va desvelando cada vez un poco más, sino que ocurre como en una verdadera conversación, que surge algo que ninguno de los interlocutores abarcaría por sí sólo ${ }^{35}$.

Esto es lo que es importante retener, y no sólo para las ciencias del espíritu, sino para el conjunto de la experiencia humana del mundo: que no hay un ser en sí, autónomo, independiente, que ya esté ahí delante y que pueda ser alcanzado o no por un sujeto cognoscente que ha de salir del ámbito de la conciencia para acceder al mundo y adecuarse al objeto o para constituir el objeto. Lo que más bien hay es una comprensión, que es interpretación porque éste es nuestro modo de ser en el mundo.

Gadamer sabe bien que en la ciencia moderna tal idea metafísica de pertenencia no tiene legitimación, pero también está persuadido de que esa misma ciencia no ha renegado totalmente de su origen griego. Por tanto, es necesario apartarnos de la fundamentación cartesiana de la ciencia moderna y resucitar algunos momentos de verdad en el pensamiento griego ${ }^{36}$. Porque los griegos

[31] Gadamer, H.-G., Verdad y método, op. cit., p. 550.

[32] Cf., Ib., p. 546.

[33] Ib., p. 549.

[34] Ib., p. 552.

[35] Ib., p. 553.

[36] Cf., Ib., pp. 550-2.

THÉMATA. Revista de Filosofía, №50 julio-diciembre (2014) pp.: 229-245 doi: 10.12795/themata.2014.i50.11 
"no intentaron fundamentar la objetividad del conocimiento desde la subjetividad y para ella. Al contrario, su pensamiento se consideró siempre desde el principio como un momento del ser mismo. (...). La dialéctica, ese antagonista del logos, no será para los griegos, como ya hemos dicho, un movimiento que lleva a cabo el pensamiento, sino el movimiento de la cosa misma que aquél percibe" ${ }^{37}$.

La identidad de pensar y ser prohíbe cualquier ejercicio de violencia metódica y exige que el pensamiento se deje guiar por el hacer de la cosa misma. "Por supuesto [precisa Gadamer] que la cosa no anda su camino ni sigue su curso sin que nosotros pensemos, pero pensar quiere decir precisamente desarrollar una cosa en su propia consecuencia" ${ }^{38}$.

En definitiva, podemos concluir que la idea gadameriana de pertenencia quiere, por una parte, restituir la auténtica dimensión ontológica de la comprensión y que, por otra parte y al hacerlo, quiere anular las limitaciones y equívocos a los que dan lugar los intentos de interpretación y "captación" de lo que es en términos sujeto-objetualistas. Porque estos son, ya se ha subrayado, los que da lugar a la eterna disputa entre realismo e idealismo y los que sin duda exigen una toma de postura anti-realista que, al mismo tiempo, sea anti-idealista. Y es que, "cuando rebasamos el concepto del objeto y de la mutua objetividad de la comprensión en dirección a una mutua pertenencia de lo subjetivo y de lo objetivo, nos limitamos a guiarnos por la necesidad de las cosas" 39 .

\subsection{La articulación griega de la pertenencia: physis y lógos}

Arraigado siempre en la filosofía griega, de la que bebió constantemente su pensamiento, Gadamer reivindica las nociones correlativas de physis y logos -donde rige la pertenencia-. A juicio de Gadamer el término physis, su significado y completa constelación de sentido es fundamental no sólo para hacernos cargo de la concepción griega de la ciencia sino, ante todo, para aproximarnos a una idea más acertada de nuestro ser en el mundo y de nuestro tratar, también teórico, en su caso, con las cosas del mundo. Nuestro pensador entiende la physis como una "existencia que se muestra y está reglamentada por sí misma", en oposición al constructivismo moderno, aquél que tiene su centro en la idea de "objeto". Al confrontar e pensamiento moderno con la herencia griega, el autor de Verdad y método, se plantea y nos plantea una pregunta. Se trata de saber si la diferencia entre la Edad Moderna y la Antigua no se podría expresar con una sola palabra: "objeto" ${ }^{40}$. Porque, a su parecer, en

[37] $I b .$, p. 551.

[38] Ib., pp. 555-556.

[39] Ib., p. 552.

[40] Cf., Gadamer, H.-G., "La filosofía griega y el pensamiento moderno", en Hermenéutica, estética e historia. Antología, Sígueme, Salamanca, 2013, p. 257.

THÉMATA. Revista de Filosofía, №50 julio-diciembre (2014) pp.: 229-245 doi: 10.12795/themata.2014.i50.11 
contraposición a la supremacía teórica del objeto, rige la reivindicación de un mundo constituido por y comprendido desde los prágmata; se trata, de nuevo, de un mundo que aparece en movimiento -ontología dinámica- y que frente a la enajenación de la objetividad clásica, aparece de modo inmediato. Y es que, el ser humano vive habitualmente en una comprensión inmediata que tiene que ver con aquello en lo que está implicado y que no se le aparece como algo enfrentado -ob-jectum-, sino como algo en medio de lo cual se mueve y cono lo que tiene que hacer algo. Sin embargo, "en el dominio moderno del mundo, un dominio estructurado por la ciencia" ${ }^{41}$ esa orientación primera e inmediata ha quedado marginada ${ }^{42}$.

Por lo que se refiere al lógos -que, entendido a modo griego, presupone un grandioso autoolvido de la conciencia, como ha subrayado Gadamer-, su recuperación proporciona la posibilidad de una comprensión no adecuacionista de la verdad. Al parecer de nuestro pensador, los griegos veían en la conciencia de sí mismo un fenómeno secundario, frente a la apertura del mundo en el que ya siempre estamos como en casa ${ }^{43}$, que es el mundo interpretado y que es el único y posible mundo. Todo el pensamiento griego constituye un "grandioso autoovido" que, posibilitado por su apertura, constituye una diferente forma de saber. Esa que "junto al gran monólogo de la ciencia moderna, de su creciente acopio de potencial de experiencias, sigue representando la otra mitad de la verdad. Y, así, el tema de la confrontación de la idea de la ciencia moderna con la idea griega de la filosofía posee una permanente actualidad ${ }^{44}$.

Y es que, posiblemente el debate en torno a la alternativa realismo-idealismo tenga su origen en la ciencia moderna y en su manera de concebirla. No es un debate que surja de manera inmediata en el mundo de la vida en el que primariamente todos somos y estamos instalados. Como ya Heidegger destacó, la pregunta por el mundo exterior es una pregunta sin sentido, porque ya todos estamos de antemano en el mundo en el que somos y existimos. Se trata por tanto de una pregunta derivada, teórica y secundaria. Esto no significa, sin embargo, que ni Heidegger ni Gadamer hayan adoptado en ningún momento una postura realista, puesto que en su punto de partida ambos se sitúan fuera de esta discusión artificial. Ni la postura realista ni la idealista se sostienen, al menos desde una concepción hermenéutico-fenomenológica, de acuerdo con lo cual, como ya se ha subrayado, ser es aparecer. Y el aparecer del fenómeno es siempre un "mostrarse ante" que reclama por tanto, para ser posible, al lógos. Pero no de manera que podamos establecer una dinámica sujeto-objetualista a partir de esta observación. De forma, por el contrario, que ya estemos siem-

[41] Gadamer, H.-G., Ib., p. 258.

[42] Cf., Ib., p. 258.

[43] Ib., p. 260.

[44] Ib., p. 261.

THÉMATA. Revista de Filosofía, Nº50 julio-diciembre (2014) pp.: 229-245 doi: 10.12795/themata.2014.i50.11 
pre y de antemano en ese mundo que comprendemos e interpretamos; ese que es mundo precisamente porque lo comprendemos e interpretamos. Pero no de manera teórica sino existencial. El ideal de la ciencia moderna -fundada en Descartes- exige un distanciamiento "artificial" de ese mundo en el que somos y así de nosotros mismos. Tal distanciamiento reclama una extrema conciencia de sí, una entronización de la subjetividad que choca frontalmente con el característico y, al decir de Gadamer, grandioso autoolvido" que el pensamiento griego tuvo de sí mismo.

En síntesis, cabe decir que la reivindicación gadameriana de physis y lógos hay inmediatamente una crítica a la ciencia moderna y a su ideal del método, pero mediatamente hay también una diferente concepción de la realidad que permite superar las limitaciones del realismo sin incurrir en los excesos del idealismo. En el lógos que se olvida de sí y se abre a los fenómenos que aparecen, a la physis, y en la physis, que no es nada distinto del mostrarse ante el lógos ya estaba operativa la pertenencia, esa que el autor de Verdad y método reivindicó en su momento.

\section{Conclusiones}

La ontología fenomenológico-hermeneútica de Gadamer se podría calificar de "dinámico-histórica", ya que entiende la realidad como un acontecer temporal. Como ya se ha indicado, la noción central desde la que se articula la posición anti-realista de Gadamer es la de pertenencia. Dicha noción es, por lo demás, incompatible con el relativismo, que no se dice igual que el perspectivismo.

$\mathrm{Al}$ evidenciar que nuestro comprender e interpretar y que nuestro inteligir está gobernado por los prejuicios, entendidos como anticipaciones de sentido; al poner de manifiesto que toda pretensión de verdad es histórica, tomamos conciencia de nuestro estar expuestos a los efectos de la historia, sabiendo que estos forman parte de nuestro ser. Entendemos entonces que los supuestos "objetos", también los de las ciencias de la naturaleza, no son independientes del lenguaje, los valores y las expectativas de quienes los investigan. Advertimos que no hay algo así como un "objeto puro", subsistiendo con independencia de nuestra inserción en el mundo histórico; que es ingenuo creer en la existencia de puros hechos, es decir, que siempre hay interpretación. Esto no significa, sin embargo, que las cosas no se muestren, significa tan sólo que hay una previa donación de sentido que es la que posibilita su mostrarse y el cómo de ese mostrarse. Es decir, el sentido es previo a cualquier hecho u objeto. No hay pues entidades objetivamente independientes de la percepción del sujeto. Aunque más bien habría que decir, "independientes de nuestra comprensión", pues ya hemos visto que no algo así como un sujeto autónomo independiente de los objetos. Y es que al eliminar el objeto eliminamos por lo mismo al sujeto. De este modo, por fin, la concepción representacionista de la verdad pierde toda credibilidad. 
Si existir es comprender y la comprensión es siempre anterior, la verdad se encuentra y da en un nivel ontológico previo al conocimiento o a la intelección. Nuestro modo inmediato de ser y estar en el mundo condiciona dicha intelección. Por esta razón el modelo moderno sujeto-objeto resulta insuficiente y perturbador, puesto que impide mirar más atrás y más al fondo; ahí donde se encuentran las condiciones de posibilidad de la experiencia, del mundo. La idea de pertenencia destaca que lo primero es la relación, la co-pertenencia de physis y lógos. Por tanto, que no hay un ser en sí, ni un sujeto autónomo y separado. De este modo zanja el debate. 
